\title{
Influence of Dextransucrase of Weissella Cibaria Nitcsk4 on Low Molecular Weight Dextran Yield: a Statistical Approach using Mixed Level Taguchi Design and Artificial Neural Network
}

\author{
Kanimozhi J., Sivasubramanian V., Ganesh Moorthy I., R. Sivashankar
}

\begin{abstract}
In the present study, the influence of dextransucrase of Weissella cibaria NITCSK4 (DSWc4), sucrose concentration, and reaction temperature on the yield of low molecular weight dextran (LMWD-DexWc4) was investigated using mixed level Taguchi design and back propagation neural network (BPNN). $B P N N$ model with three neurons in a hidden layer generated a low mean squared error (MSE). The determination coefficients $\left(R^{2}\right.$ -value) for ANN and Taguchi models were 0.991 and 0.998 , respectively. Considering absolute average deviation (AAD) and MSE, Taguchi model is more adequate. Among three factors, the percentage yield of low molecular weight of dextran is invariably dependent on the sucrose concentration. The study suggested that a low sucrose concentration (3\% w/v), DSWc4 $(0.25 \mathrm{IU} / \mathrm{ml})$ and slightly high temperature $\left(35^{\circ} \mathrm{C}\right)$ ultimately favored the production of LMWD-DexWc4 (91.639\%). LMW-DexWc4 produced by DSWc4 at optimized conditions was analyzed. The weight average molecular weight of LMW-DexWc4 was calculated using $\mathrm{M}-\mathrm{H}$ expression, found to be $85775(\approx 90 \mathrm{kDa})$. The relative percentage error between the number and weight average molecular weight was found to be less (4.42\%). The polydispersity $(P D)$ index of the LMW-DexWc4 was found to be 0.9576 and the value is close to 1 . The PD value depicted that the molecular weight distribution of dextran was narrowly dispersed.
\end{abstract}

Keywords: Taguchi Orthogonal Array, ANN, Dextran, Dextransucrase, Weissella cibaria NITCSK4.

\section{INTRODUCTION}

Dextran is an FDA (Food and Drug Administration) approved bacterial extracellular polymeric carbohydrate composed of long chains of glucose units connected mainly by $\alpha-(1-6)$ linkage. Dextran are often preferred over synthetic polymers due to their solubility in water, non-toxic, low cost, availability, biocompatible and biodegradable. Dextrans are widely used in food, pharmaceutical, and chemical industries as adjuvant, emulsifier, carrier and stabilizer. Rather a native dextran, low molecular weight (LMWD) dextran serve as one

Revised Manuscript Received on December 15, 2019.

* Correspondence Author

Kanimozhi J., School of Bio and Chemical Engineering, Department of Biotechnology, Kalasalingam Academy of Research and Education Krishnankoil, India. kanimozhireddy@yahoo.co.in

Sivasubramanian V.*, Department of Chemical Engineering, National Institute of Technology Calicut, Kozhikode, Kerala, India. siva@ nitc.ac.in

Ganesh Moorthy I., Department of Biotechnology, Kamaraj College of Engineering and Technology, Virudhunagar, Tamilnadu, India. igmoorthy@yahoo.co.in

R. Sivashankar, Department of Chemical Engineering, Hindustan Institute of Science and Technology, Chennai, India. rsivasankhar@ymail.com of the most promising macromolecular carrier material for a wide variety of therapeutic agents. LMWD is used medicinally as an antithrombotic (antiplatelet) to reduce blood viscosity and as a volume expander in anemia. Native dextran with a molecular weight of about $5 \times 10^{8} \mathrm{Da}$ is unsuitable as a blood plasma substitute. In general, the ideal size for blood plasma dextran, so-called clinical dextran, is $40-100 \mathrm{kDa}$ Rheology of native dextrans in relation to their primary structure [1]. In addition, the therapeutic components with too low molecular weight are rapidly lost from the circulation by removal via the kidneys. Thus, the isomalto oligodextrans are therapeutically ineffective. Conversely, the material with too high molecular weight can interfere with normal coagulation processes of the blood and/or with greater properties of non $\alpha-(1-6)$ linkage and causes a greater incidence of allergic reactions.

Clinically low molecular weight dextran ranges from 5.0 $\mathrm{kDa}$ to $100 \mathrm{kDa}$ is preferred than high molecular weight dextran. In general, the industries are preparing LMW (clinical dextran) dextran by adopting different strategies which include conventional ethanol fractionation followed by degradation of native dextran molecules such as acid hydrolysis, enzymolysis (dextranase), ultrasonication, and shear degradation. The uses of Lipomyces starkeyi, Aspergillus wentii, and Penicillium sp have been reported for the LMWD preparation. Though, the above strain produces dextranase, an enzyme that cleaves dextran into smaller fragments. Either two-stage fermentation process or mixed culture system is adopted with Lipomyces for the low molecular weight dextran production. In alternative process, the acceptor (secondary carbohydrate) molecules are incorporated in fermentation media to limit the molecular size of dextran [1]-[5]. In addition, a reactor of special membrane type is used to prepare low molecular weight dextran in order to lower the production cost by limiting downstream operations. In 2002, Mountzouris and his team produced oligodextrans by hydrolyzing high molecular weight dextran using endodextranase [6]. Though, the conventional ethanol fractionation methods are more laborious and time-consuming, and ultrafiltration technique also has its limitation.

Published By:

Blue Eyes Intelligence Engineering 
Apart from the process variables influence of dextran, molecular weight, branching, and yield were comprehensively studied by dextransucrase of $L$. mesenteroides B512 (f). In 2003, Kim and group extensively studied the effect of sucrose and temperature on the molecular weight distribution of dextran by dextransucrase of $L$. mesenteroides B512 (F) [7]. As per the earlier study, the dextran structure and molecular mass are depend on sucrose concentration, dextransucrase concentration, and process temperature [8]. Owing to the limitations and cost of downstream processes of hydrolysis of native dextran, the study focused on the conventional enzymatic conversion of sucrose into LMWD. The strain NITCSK4 (KX387868) exhibited highly viscous linear dextran (DexWc4-dextran of Weissella cibaria NITCSK4) by the catalytic conversion of dextransucrase (162 kDa) [9]. The water-soluble linear DexWc4, has a biodegradable nature, it can be deliberately used for human. However, the native DexWc4 with $4.2 \times 10^{4}$ $\mathrm{kDa}$ molecular weight is not suitable in medical context because of its immunogenicity in the bloodstream. As reported earlier, the molecular weight of dextran also depends on the dextransucrase concentration. Therefore, the molecular weight of dextran cannot be controlled in microbial fermentative production. The present work is intended for the enzymatic conversion of sucrose into LMWD by dextransucrase of NITCSK4 (DSWc4). The enzymatic reaction condition is controlled to prepare LMWD which is expected to cut the hydrolysis of native dextran. The yield of dextransucrase activity virtuously depends on the effect of sucrose, temperature, and dextransucrase concentration on the yield of LMWD is intended. However, the correlations between the yield and molecular weight distribution have not been studied earlier. Thus, the work is set based on mixed level Taguchi orthogonal array design instead of adopting the conventional OVAT approach. Since the Taguchi design could be used to comprehend the factors involved in the process [10]. The artificial neural network (ANN) with feed forward back propagation is adopted to find the influence of sucrose, dextransucrase and temperature on the yield of LMWD. The catalytic reaction mechanism of dextran synthesis by two site insertion reaction mechanism is considered [5] for the evaluation of Michaelis Menton kinetic parameters $\left(K_{\mathrm{M}}\right.$ and $\left.\mathrm{V}_{\max }\right)$.

\section{MATERIAL AND METHODS}

\section{A. Chemicals and Reagents}

All reagents and chemicals were purchased from Merck chemicals and Sigma chemical co. The strain Weissella cibaria NITCSK4 was isolated from the deteriorated sugarcane juice. Membrane filters $0.45 \mu$ and $0.2 \mu$ were purchased from Millipore. Disodium 2, 2'-bicinchoninate, L-serine, and sephacryl-S-300HR were purchased from Sigma Chemical Co (USA). Standard dextran (10, 70, $5000-40000 \mathrm{kDa})$, blue dextran (2000 kDa), sucrose, sodium carbonate monohydrate, sodium bicarbonate, copper sulphate, were purchased from Himedia Pvt. Ltd. India.

\section{B. DSWc4 Production and Determination of Dextransucrase Activity}

The synthesis of DSWc4 was induced by incorporating 3\% (w/v) sucrose in MRS media (with $0.2 \%$ glucose) by Weissella cibaria NITCSK4. The fermentation was carried out for $10 \mathrm{~h}$ at $26^{\circ} \mathrm{C}$. After fermentation, the bacterial cells were pelleted by centrifugation at $7000 \mathrm{rpm}$ for $20 \mathrm{~min}$ at $4^{\circ} \mathrm{C}$. The bacterial culture supernatant was filtered through $0.2 \mu$ membrane filter. The proteins present in the cell-free culture filtrate were precipitated with $35 \%$ of polyethylene glycol (PEG-400) and incubated at $4{ }^{\circ} \mathrm{C}$ for overnight $(16 \mathrm{~h})$. The precipitates were separated by centrifugation $(20000 \mathrm{rpm}$ at $4^{\circ} \mathrm{C}$ for $\left.30 \mathrm{~min}\right)$. The pellets were suspended in $0.02 \mathrm{M}$ sodium acetate buffer ( $\mathrm{pH} 5.5)$ and dialyzed (14 kDa cut off) against sodium acetate buffer. The crude concentrate was used for the synthesis of dextran. The crude dextransucrase concentrate activity was determined by the assay as described in [11]. The activity unit (IU) is described as the amount of dextransucrase catalyzing the formation of $1 \mu \mathrm{mol}$ of $\mathrm{d}$-fructose from reaction mixture sucrose $(0.2 \mathrm{M})$ in acetate buffer $(0.05 \mathrm{M}, \mathrm{pH} 5.5)$ containing $\left.\mathrm{CaCl}_{2}(0.18 \mathrm{M})\right]$ at $25^{\circ} \mathrm{C}$ in one minute.

\section{Taguchi Orthogonal Array}

The influence of dextransucrase of Weissella cibaria NITCSK4 (DSWc4), sucrose concentration and temperature on the yield of LMW-DexWc4 was investigated using mixed level Taguchi design. The Taguchi orthogonal array $\left(\mathrm{L}_{18}\right)$ mixed level $\left(\mathrm{L}_{18}-6^{1} \times 3^{2-1}\right)$ fractional factorial design: sucrose concentration at six levels and enzyme concentration and temperature at 3 levels (18 runs) were created using Minitab 16 software. The design factors and their levels were chosen and set. The factors and their levels considered for enzymatic conversion of dextran are shown in Table I.

TABLE-I: Variables with their Different Levels Applied In Taguchi $\left(\mathrm{L}_{18}\right)$ Orthogonal Array

\begin{tabular}{|c|c|c|c|}
\hline \multirow{2}{*}{ Levels } & \multicolumn{3}{|c|}{ Variables } \\
\cline { 2 - 4 } & Sucrose & $\begin{array}{c}\text { Dextransucrase } \\
\text { (NITCSK4) } \\
\boldsymbol{N} / \boldsymbol{v}\end{array}$ & Temperature \\
\hline 1 & 3 & 0.125 & ${ }^{\circ} \boldsymbol{C}$ \\
\hline 2 & 6 & 0.250 & 25 \\
\hline 3 & 9 & 0.375 & 35 \\
\hline 4 & 12 & - & 45 \\
\hline 5 & 15 & - & - \\
\hline 6 & 18 & - & - \\
\hline
\end{tabular}

$*_{1} I U=\mu M$ of fructose $/ \mathrm{mL} \mathrm{min}^{-1}$

The study intended to study the main influences of sucrose, temperature and enzyme concentration. However, the interactions among the mentioned factors are assumed to be negligible. In Taguchi method, the experimental results were converted into Signal/Noise $(\mathrm{S} / \mathrm{N})$ ratio to compute the quality uniqueness differing from the desired value. The high value of $\mathrm{S} / \mathrm{N}$ ratio directly implicit the significant parameters [12]. 
The $\mathrm{S} / \mathrm{N}$ ratio calculation depends on each run of the experiment on higher-the-better percentage yield of LMW-DexWc4. The S/N ratio was calculated to ascertain the most robust set of process conditions within the outcomes from the equation (1).

$$
\frac{S}{N}=-10 \log _{10} \frac{1}{n} \sum_{i=1}^{n} \frac{1}{y_{i}^{2}}
$$

where $y$ is the signal and $n$ is the number of repetitions in each experiment and $y$ is the observed yield percentage of LMWD in a run. The experimental percentage yield of LMWD acquired was evaluated using Minitab 16 software to assess the effect of individual factors.

\section{Enzymatic Conversion of Sucrose into Dextran}

The crude enzyme was designated as DSWc4 and used for the conversion of sucrose into dextran. Briefly, $0.05 \mathrm{M}$ sodium acetate assay buffer ( $\mathrm{pH} 5.5$ ) was prepared with $0.18 \mathrm{M} \mathrm{CaCl}_{2}$. The $100 \mathrm{~mL}$ of the reaction mixture was prepared with $5 \mathrm{ml}$ of crude enzyme concentrate $(8 \mathrm{IU} / \mathrm{ml})$ at three different levels $12.5,25,37.5 \mathrm{IU}$ (total enzyme concentration in $100 \mathrm{~mL}$ ) and $95 \mathrm{ml}$ of $0.05 \mathrm{M}$ sodium acetate buffer with varied sucrose concentration at six levels from $3 \%(\mathrm{w} / \mathrm{v})$ to $18 \%$ of sucrose. The enzymatic conversion of sucrose to glucan was performed at three different temperatures 25,35 , and $45^{\circ} \mathrm{C}$ with stirring at $200 \mathrm{rpm}$ for $24 \mathrm{~h}$. $20 \mathrm{~mL}$ of aliquots of the reaction mixture were adjusted to $100 \mathrm{~mL}$ and boiled for 10 min to inactivate the enzyme and to liberate enzyme bound product. The dextran (DexWc4) from the reaction mixture was separated by ice-cold ethanol precipitation. The dextran yield was quantified by the Phenol-Sulphuric acid method.

\section{E. Recovery and Analysis of Dextran}

After conversion, the polysaccharide was precipitated with double the amount of chilled ethanol and then the precipitate was suspended in deionized water. The precipitation cycles were repeated for 3 times. The powder form of polysaccharide was prepared by lyophilisation. The molecular mass of dried polysaccharide was analyzed by gel permeation chromatography (GPC). The dextran was fractionated based on their size by GPC using sephacryl-S-300HR. The glass column $\left(201 \mathrm{~cm}^{2}\right.$ cross-sectional area) with the height of $30 \mathrm{~cm}$ was packed with sephacryl-S-300HR slurry suspension in $0.05 \mathrm{M}$ citrate buffer. The sample $(2 \mathrm{mg} / \mathrm{mL})$ was applied through a glass column packed and eluted with citrate phosphate buffer $(0.05 \mathrm{M}, \mathrm{pH} 5.0)$ at a flow rate of $1 \mathrm{~mL} / \mathrm{min}$. The fractions $(1$ $\mathrm{mL} / \mathrm{min}$ ) were collected and absorbance was read at $620 \mathrm{~nm}$ [10]. The elution pattern of DexWc4 was compared with standards such as dextran $40 \mathrm{kDa}, 70 \mathrm{kDa}$, and $100 \mathrm{kDa}$. The fractions $<100 \mathrm{kDa}$ were collected and pooled together and number average molecular weight (MWn) was determined by the Copper-Bichinchonate $(\mathrm{Cu}-\mathrm{Bic})$ method. The amount of dextran pooled GPC fractions was quantified by the Phenol-Sulphuric acid method. The percentage yield of LMWD was calculated by the equation (2):

$$
\operatorname{LMWD}(\%)=\frac{x}{y} \times 100
$$

where as $\mathrm{x}$ is the amount of Low molecular weight dextran in $\mathrm{mg} / \mathrm{mL}$ (GPC fractions) and $\mathrm{y}$ is the total amount of dextran in $\mathrm{mg} / \mathrm{mL}$.

\section{F. Analysis of Variance (ANOVA)}

The ANOVA was performed to analyze the statistical significance of the low molecular weight dextran production. The results were interpreted to conclude the influence of process parameters on low molecular weight dextran yield. The reaction conditions of the process parameters were predicted. Finally, the predicted conditions were experimentally validated.

\section{G. Artificial Neural Network (ANN)-Feed Forward Back Propagation}

The most common model to study the non-linear mapping among the variables (input and output) is back propagation neural network (BPNN). The feed forward BPNN (nntool in MATLAB version 7.6.0.324 R2008a) was preferred though, the algorithm back propagated by adjusting the weights to minimize the error difference between the target output and predicted output. The experimental yield percentage obtained from the Taguchi design experiments were used to frame effective ANN model [13]. Thus, the error between observed and predicted output unit will be minimized. Out of 18 runs, $70 \%$ of the data were trained, $15 \%$ data were validated and $15 \%$ data were tested with a neural network. The network was trained with Levenberg-Marquardt function, Weight/bias learning function-Gradient Descent with momentum, single hidden layer with different hidden neurons and TANSIG transfer functions, Linear output transfer function PURELIN (Fig. 1)

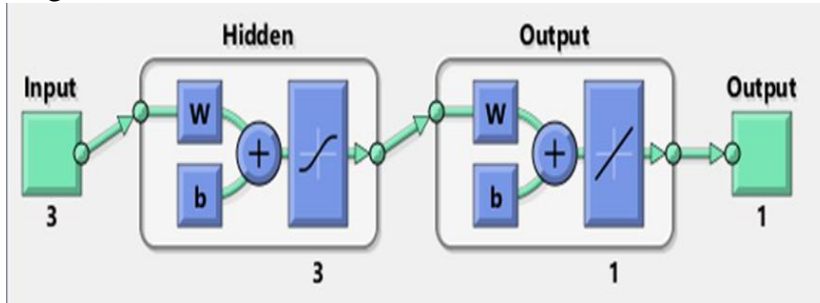

Fig .1 BPNN architecture used for optimization of percentage yield of LMWD.

The impact of number of neurons in hidden layer was analyzed to find an optimum number of neurons by calculating minimum mean squared error (MSE) and coefficient of determination $\left(\mathrm{R}^{2}\right)$.

\section{H. Validation of the Model and Analysis of LMWD}

The LMWD was produced by enzymatic conversion (DSWc4 $5 \mathrm{IU} / \mathrm{ml}$ ) of sucrose $(3 \% \mathrm{w} / \mathrm{v})$, at $35^{\circ} \mathrm{C}$ for $24 \mathrm{~h}$. The dextran concentration was quantified by the Phenol-sulphuric acid method. The dextran was precipitated and fractioned by gel permeation chromatography as described earlier. The GPC fractions absorbance $\left(\mathrm{A}_{620 \mathrm{~nm}}\right)$ was measured. The dextran in the fractions $(<100 \mathrm{kDa})$ was combined together and designated as LMW-DexWc4. The LMW-DexWc4 was precipitated and freeze-dried. The number average molecular weight of LMW-DexWc4 was determined by the $\mathrm{Cu}-\mathrm{Bic}$ method 
The structural morphology of LMW-DexWc4 was analyzed by SEM and compared with the standard dextran (70 $\mathrm{kDa}$ ). Further, the viscosity of LMW-DexWc4 was measured with Ostwald viscometer. The capillary viscometer was used to determine the relative viscosity of the very dilute LMW-DexWc4 solution and solvent (water) at $30^{\circ} \mathrm{C}[14]$. The relative viscosity $\left(\eta_{\mathrm{r}}\right)$ and inherent viscosity $\left(\eta_{\mathrm{i}}\right)$ of dextran solution were calculated as described in equation (3) and (4): The LMW-DexWc4 solution viscosity $(\eta)$ is related to the solvent viscosity $\left(\eta_{\mathrm{o}}\right)$, resulting in the relative viscosity. $\eta_{r}=\frac{\eta}{\eta_{0}}=\frac{t \rho}{t_{0} \rho_{0}}$

where $\eta$ (poise) is the viscosity, $\rho$ and $\rho_{0}$ density of dextran solution and water $\left(\mathrm{g} / \mathrm{cm}^{3}\right)$ and $\mathrm{t}, \mathrm{t}_{0}$ the liquid flow draining time (seconds) of dextran solution and water respectively.

$\eta_{i}=\eta_{r}-1$

The intrinsic viscosity [ $\eta]$ was calculated by the Solomon-Ciuta single-point equation (5)

$[\eta]=\frac{1}{c} \sqrt{2 \eta_{i}-2 \ln \eta_{r}}$

The relation between molecular weight $\mathrm{M}_{\mathrm{w}}$ and the intrinsic viscosity is specified by Mark-Houwink equation [14]

$[\eta]=\kappa_{M H}\left(M_{w}\right)^{a}$

where $\mathrm{k}_{\mathrm{MH}}\left(\mathrm{cm}^{3} / \mathrm{g}\right)$ and $a$ are Mark-Houwink constants.

\section{RESULTS AND DISCUSSIONS}

\section{A. Taguchi Design Analysis}

The effect of sucrose, temperature, and DSWc4 on the percentage yield of low molecular weight dextran was studied by Taguchi's Orthogonal Array design. For three parameters and mixed levels, the $\mathrm{L}_{18}$ orthogonal array was selected. The experiments were conducted at different sucrose concentrations (3-18\% w/v), DSWc4 concentrations (2.5-7.5 $\mathrm{IU} / \mathrm{ml})$ and temperatures $\left(25-45^{\circ} \mathrm{C}\right)$. The influence of sucrose, temperature, and dextransucrase on the yield of dextran by Weissella cibaria NITCSK4 was extensively studied [9]. Thus, the purpose of the study was put on the yield of low molecular dextran (\% LMWD). Totally eighteen experiments were conducted and $\mathrm{S} / \mathrm{N}$ ratios for (\% LMWD) were analyzed. The experimentally observed and Taguchi predicted yield of \% LMWD is presented in Table II.

The analysis of variance (Table III) shown that the sucrose, DSWc4, and temperature were significant for dextran production $(\mathrm{p} \approx 0.000)$. The high $(0.998)$ regression based coefficient of determination $\mathrm{R}^{2}$ explains that the model attribute $99.8 \%$ of the variability on the yield and also describes the model significance. In addition, the coefficient of determination $\left(\mathrm{R}^{2}\right)$ value indicates the good correlation between observed and predicted values of percentage yield of LMWD. The effect of three variables, namely Sucrose, DSWc4, and temperature was studied for their significance on percentage yield of low molecular weight dextran production by Taguchi orthogonal array $\mathrm{L}_{18}$ designs. The mixed level Taguchi design showed a wide variation in LMWD yield from $8.139 \%$ to $91.639 \%$ and dextran (DexWc4) yield from 4.154

to $42.923 \mathrm{mg} / \mathrm{mL}$. It was inferred from the analysis of variance that the factors considered in Taguchi design were statistically significant at $95 \%$ confidence limit. The correlation coefficient between experimental yield with Taguchi predicted yield was 0.999 (R). The most significant factors and their levels were determined based on their $\mathrm{S} / \mathrm{N}$ ratio and delta rank. The order of influence of factors on the percentage yield of low molecular weight dextran (LMWD or LMW-DexWc4): Sucrose >Temperature > Enzyme. The influence of the factors was considered based on the percentage yield of low molecular weight dextran.

\section{TABLE-II: Dextran Yield and Percentage Yield of} LMWD

\begin{tabular}{|c|c|c|c|c|c|c|c|c|}
\hline \multirow{3}{*}{$\underset{\Xi}{\Xi}$} & \multirow{3}{*}{ 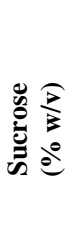 } & \multirow{3}{*}{$\overbrace{0}^{\searrow ٍ}$} & \multirow{3}{*}{ 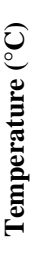 } & \multirow{3}{*}{ 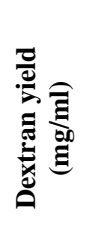 } & \multirow{3}{*}{ 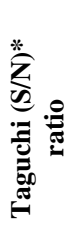 } & \multicolumn{3}{|c|}{ LMWD (\%) } \\
\hline & & & & & & \multirow[b]{2}{*}{$\operatorname{Exp}$} & \multicolumn{2}{|l|}{ Predicted } \\
\hline & & & & & & & Taguchi & $A N N^{\#}$ \\
\hline 1 & 1 & 1 & 1 & 14.43 & 37.65 & 76.30 & 76.61 & 77.00 \\
\hline 2 & 1 & 2 & 2 & 12.25 & 39.24 & 91.63 & 91.27 & 91.51 \\
\hline 3 & 1 & 3 & 3 & 4.15 & 38.38 & 83.05 & 83.11 & 81.18 \\
\hline 4 & 2 & 1 & 1 & 25.35 & 36.56 & 67.30 & 68.61 & 68.01 \\
\hline 5 & 2 & 2 & 2 & 23.69 & 38.70 & 86.13 & 83.27 & 82.41 \\
\hline 6 & 2 & 3 & 3 & 5.62 & 37.33 & 73.55 & 75.11 & 76.49 \\
\hline 7 & 3 & 1 & 2 & 29.30 & 34.89 & 55.55 & 56.11 & 58.44 \\
\hline 8 & 3 & 2 & 3 & 6.04 & 35.38 & 58.80 & 58.61 & 58.96 \\
\hline 9 & 3 & 3 & 1 & 34.74 & 34.26 & 51.63 & 51.27 & 52.32 \\
\hline 10 & 4 & 1 & 3 & 6.13 & 21.74 & 12.22 & 11.44 & 19.47 \\
\hline 11 & 4 & 2 & 1 & 39.95 & 22.20 & 12.88 & 14.78 & 11.31 \\
\hline 12 & 4 & 3 & 2 & 34.01 & 25.97 & 19.88 & 18.78 & 19.38 \\
\hline 13 & 5 & 1 & 2 & 37.48 & 19.13 & 9.05 & 10.11 & 14.83 \\
\hline 14 & 5 & 2 & 3 & 5.87 & 20.63 & 10.80 & 12.61 & 12.94 \\
\hline 15 & 5 & 3 & 1 & 42.93 & 18.21 & 8.13 & 5.28 & 9.74 \\
\hline 16 & 6 & 1 & 3 & 5.73 & 19.60 & 9.55 & 7.11 & 12.64 \\
\hline 17 & 6 & 2 & 1 & 40.80 & 20.60 & 10.72 & 10.44 & 9.68 \\
\hline \multirow[t]{5}{*}{18} & 6 & 3 & 2 & 35.73 & 21.38 & 11.72 & 14.44 & 11.75 \\
\hline & & & & & & AAD & 0.460 & 9.80 \\
\hline & & & & & & MSE & 2.469 & 7.95 \\
\hline & & & & & & $\mathbf{R}$ & 0.999 & 0.996 \\
\hline & & & & & & $\mathbf{R}^{2}$ & 0.998 & 0.991 \\
\hline
\end{tabular}

Published By: 
The analysis of variance (Table III) shown that the sucrose, DSWc4, and temperature were significant for dextran production $(\mathrm{p} \approx 0.000)$. The high $(0.998)$ regression based coefficient of determination $\mathrm{R}^{2}$ explains that the model attribute $99.8 \%$ of the variability on the yield and also describes the model significance. In addition, the coefficient of determination $\left(\mathrm{R}^{2}\right)$ value indicates the good correlation between observed and predicted values of percentage yield of LMWD. The effect of three variables, namely Sucrose, DSWc4, and temperature was studied for their significance on percentage yield of low molecular weight dextran production by Taguchi orthogonal array $\mathrm{L}_{18}$ designs. The mixed level Taguchi design showed a wide variation in LMWD yield from $8.139 \%$ to $91.639 \%$ and dextran (DexWc4) yield from 4.154 to $42.923 \mathrm{mg} / \mathrm{mL}$. It was inferred from the analysis of variance that the factors considered in Taguchi design were statistically significant at $95 \%$ confidence limit. The correlation coefficient between experimental yield with Taguchi predicted yield was $0.999(\mathrm{R})$. The most significant factors and their levels were determined based on their $\mathrm{S} / \mathrm{N}$ ratio and delta rank. The order of influence of factors on the percentage yield of low molecular weight dextran (LMWD or LMW-DexWc4): Sucrose >Temperature > Enzyme. The influence of the factors was considered based on the percentage yield of low molecular weight dextran.

TABLE-III: Analysis of Variance (ANOVA) and S/N Ratio Showing the Variables Influence on the Percentage Yield of LMWD

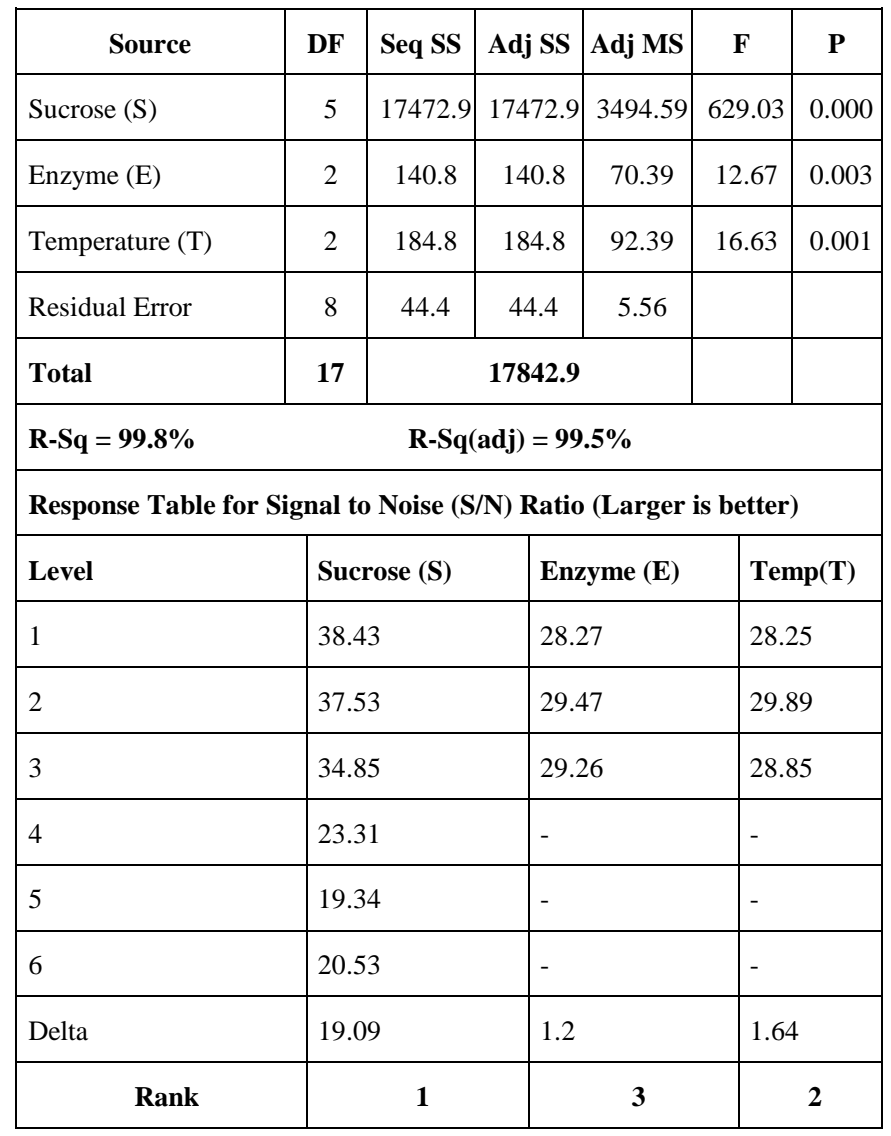

\section{B. Back Propagation Neural Network (BPNN)}

The model with three neurons in a hidden layer generated a low mean squared error (MSE) and high $\mathrm{R}^{2}$ value as shown in Fig. 2. The determination coefficients $\left(\mathrm{R}^{2}\right)$ for ANN and Taguchi models were 0.991 and 0.998 (Table 2) respectively. The goodness and correlation of the neural network prediction and Taguchi prediction with the experimental response was analyzed by calculating the $\mathrm{R}^{2}$ and AAD which was found to be low for the Taguchi prediction. Considering absolute average deviation (AAD) and MSE, Taguchi model is more adequate. The enzymatic conversion study suggested that a low sucrose concentration and slightly high temperature $\left(35^{\circ} \mathrm{C}\right)$ than the optimum temperature ultimately favors the production of low molecular weight dextran (91.639\%). In spite of the yield of low molecular weight dextran, the enzyme yielded very low total dextran.

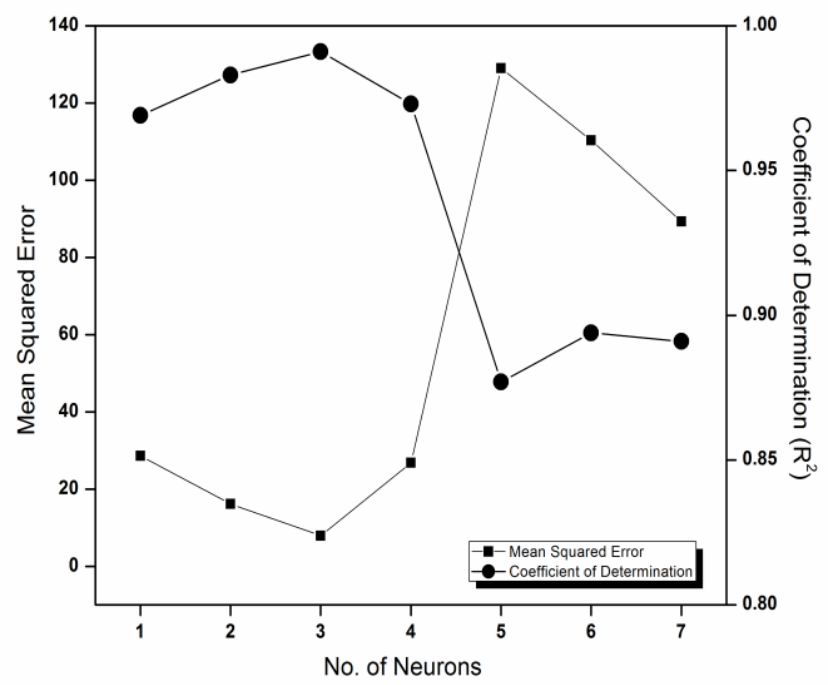

Fig. 2 Influence of number of neurons in hidden layer versus co-efficient of determination $\left(R^{2}\right)$ and mean squared error (MSE)
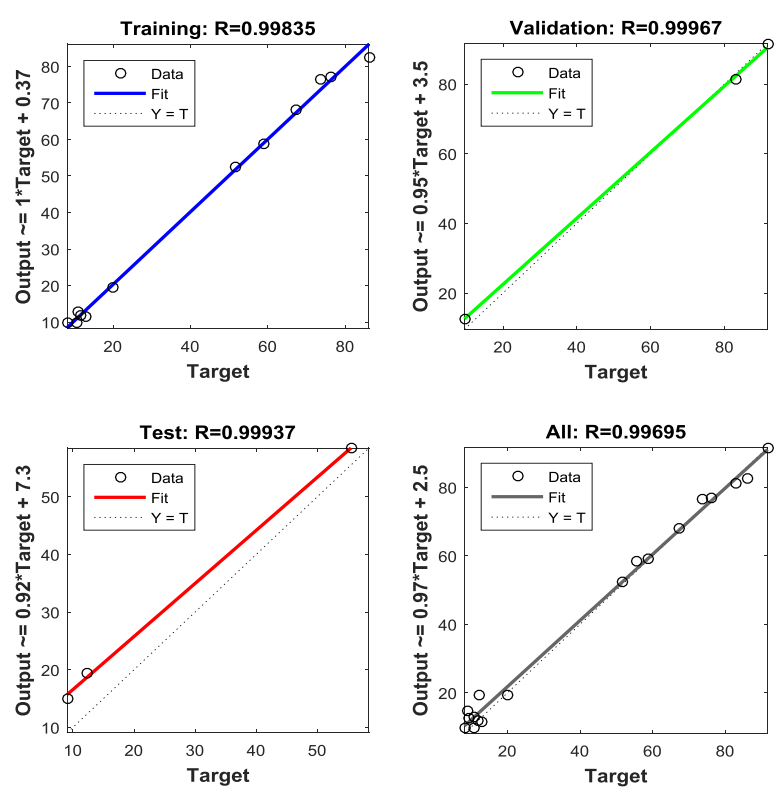

Fig. 3 Regression plot of observed and predicted percentage yield of low molecular weight dextran (\%LMWD) 
The determination coefficients $\left(\mathrm{R}^{2}\right)$ for ANN and Taguchi models were 0.991 and 0.998 (Table 2) respectively. The goodness and correlation of the neural network prediction and Taguchi prediction with the experimental response was analyzed by calculating the $\mathrm{R}^{2}$ and $\mathrm{AAD}$ which was found to be low for the Taguchi prediction. Considering absolute average deviation (AAD) and MSE, Taguchi model is more adequate. The enzymatic conversion study suggested that a low sucrose concentration and slightly high temperature $\left(35^{\circ} \mathrm{C}\right)$ than the optimum temperature ultimately favors the production of low molecular weight dextran (91.639\%). In spite of the yield of low molecular weight dextran, the enzyme yielded very low total dextran. The dextran production through microbial fermentation yielded a higher amount of native dextran than the enzymatic process. However, owing to the consideration of downstream processing of preparation of LMW-DexWc4 from native dextran, the enzymatic production process could be adapted for LMWD preparation.

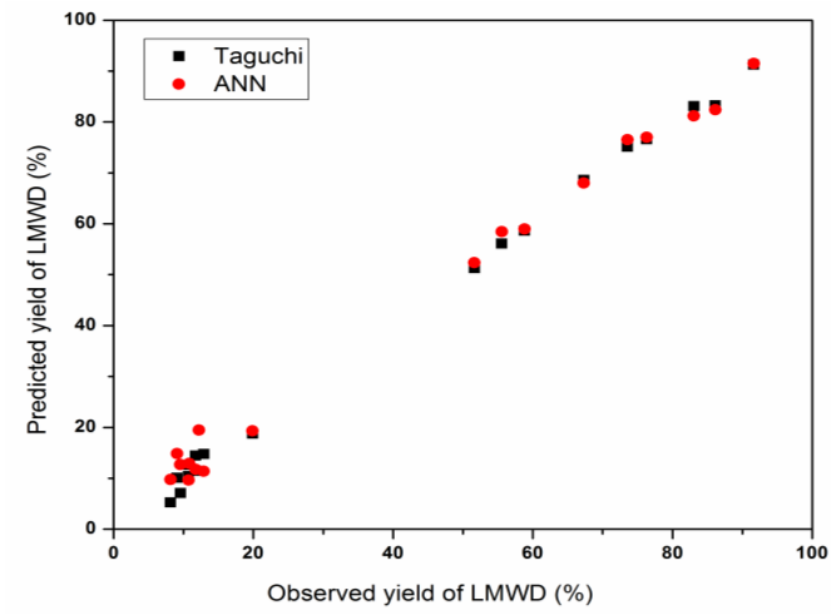

Fig. 4 Correlation of observed and predicted percentage yield of LMWD

\section{Effect of Sucrose on LMWD Yield}

The $\mathrm{S} / \mathrm{N}$ (Larger is better) ratio of the factors at their different levels were presented in Table 3. The variable sucrose at $3 \%(\mathrm{w} / \mathrm{v})$ secured maximum $\mathrm{S} / \mathrm{N}$ ratio for the percentage yield of LMW-DexWc4. Similarly, the DSWc4 at $5 \mathrm{IU} / \mathrm{ml}$, and temperature at $35^{\circ} \mathrm{C}$ has the maximum of $\mathrm{S} / \mathrm{N}$ ratio. The run order no. 2 has the maximum of $\mathrm{S} / \mathrm{N}$ ratio of 39.242 for $92 \%$ yield of LMWD at sucrose $3 \%(\mathrm{w} / \mathrm{v})$, the $\mathrm{DSWc} 4$ at $5 \mathrm{IU} / \mathrm{ml}$, and temperature at $35^{\circ} \mathrm{C}$. The Taguchi mixed level design ascertained that the molecular weight of dextran merely depends on the substrate concentration. The higher concentration of sucrose favored the greater yield of HMWD. However, the total dextran produced at run order 2 was considered to be $14.256 \mathrm{mg} / \mathrm{ml}$, out of which $92 \%$ of dextran size was $<100 \mathrm{kDa}$. Thus the concentration of sucrose determines the molecular weight of dextran (DexWc4) of Weissella cibaria NITCSK4. The effect of sucrose concentration on the low molecular weight distribution of DexWc4 was conceded to the Falconer findings. Falconer et al., 2011 studied the effect of sucrose concentration, dextransucrase concentration, and temperature on the molecular weight distribution of dextran by Leuconostoc mesenteroides B-512FMC dextransucrase. They stated that the molecular weight of the synthesized dextran increased as the concentration of sucrose was increased. Falconer studied
Michaelis-Menten kinetics of dextransucrase and ascribed that the unique deviation in molecular mass and formation of dextran, which is proportional to the utilization of the substrate (sucrose) [8]. Kim et al., (2003) concluded that the sucrose concentration and yield of high molecular weight dextran (HMWD) have an inverse relationship which was in contradict with the study of Falconer [7]-[8]. The effect of sucrose on the percentage yield of LMWD responses was stimulated with the trained ANN network model and the effect is presented in Fig. 5. The molecular weight distribution of dextran is merely depending on the sucrose.

\section{Effect of Dextransucrase concentration on LMWD Yield}

In addition, the type of substrate and the characteristics of the catalyst account for the variations in the dextran molecular weight distribution (MWD). Robyt et al. (2008) have studied the molecular weight distribution of dextransucrase using a constant concentration of sucrose $(20 \mathrm{mM})$ and varying the concentration of the enzyme $(10 \mathrm{U} / \mathrm{mL}, 1.0 \mathrm{U} / \mathrm{mL}$, and 0.1 $\mathrm{U} / \mathrm{mL}$ ) and found that the average molecular weights of the dextran is inversely proportional to the concentration of the enzyme [5]-[8]. This was inferred to be the processivity of the polymerization reaction of dextransucrase. Falconer et al. (2011) observed that the molecular weights of the synthesized dextrans were inversely proportional to the dextransucrase concentration. It was found that the number-average molecular weights were the lowest $(100 \mathrm{kDa})$ when the dextransucrase concentration was the highest $(50 \mathrm{U} / \mathrm{mL})$, demonstrating that the molecular weights of the synthesized dextran was inversely proportional to the enzyme concentration. The DSWc4 yielded higher percentage (92\%) of low molecular weight dextran $(<100 \mathrm{kDa})$ when the enzyme concentration was $0.250 \mathrm{IU} / \mathrm{mL}$ and sucrose concentration was lesser. The effect of enzyme concentration on the yield of LMWD is presented in Fig. 5; the stimulated responses have shown that the lower enzyme (DSWc4) concentration favors the yield of low molecular weight dextran at $35^{\circ} \mathrm{C}$.

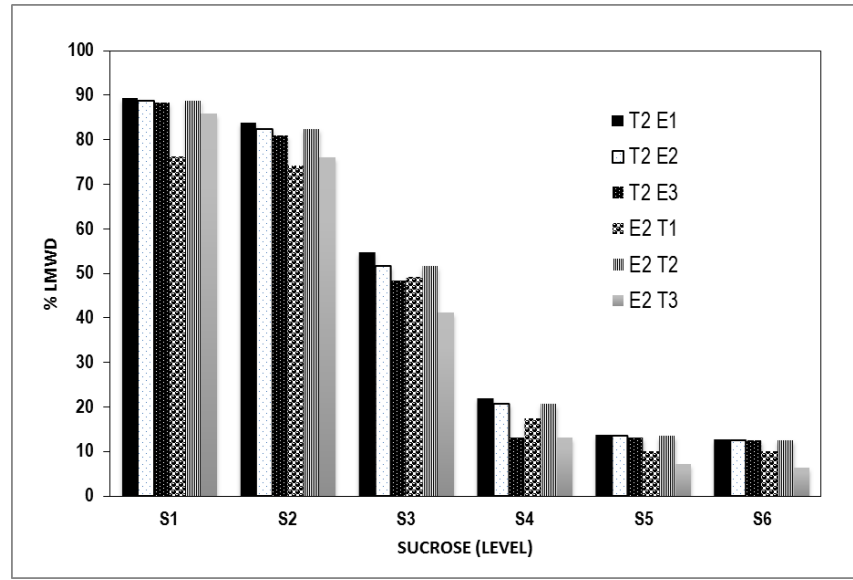

Fig. 5 Influence of sucrose, enzyme, and temperature on the production of low molecular weight dextran (\% LMWD)

\section{E. Effect of Temperature on LMWD Yield}


It has been described earlier that temperature influenced the yield, and rate of formation, and the molecular mass distribution of dextran [8]. Kim et al., (2003) showed that branching was increased from $4.8 \%$ at $4{ }^{\circ} \mathrm{C}$ to $14.7 \%$ at $45^{\circ} \mathrm{C}$ during cell-free synthesis of dextrans by a dextransucrase from $L$. mesenteroides B-512FMCM. Though the temperature and $\mathrm{pH}$ have not influenced and significant variations were observed in the molecular weight of dextran. An earlier report distinguished that the dextran produced at three different temperatures namely at 3,10 , and $20^{\circ} \mathrm{C}$ is shown an expanded conformation, while dextrans synthesized at $30^{\circ} \mathrm{C}$ were more compact [15]. As per their report, reaction temperature and molecular weight of dextran is inversely proportional [1]. In microbial fermentation, the yield of dextran by Weissella cibaria was considerably less at higher temperature $\left(35^{\circ} \mathrm{C}\right)$ as compared to the dextran production at $26^{\circ} \mathrm{C}$ [9]. The strain NITCSK4 produced high molecular weight dextran (highly viscous and linear dextran) at a lower temperature $\left(26^{\circ} \mathrm{C}\right)$.Similarly, the effect of reaction temperature on the rheological properties of native dextran has been reviewed earlier and they found that the dextran branching was not as much at lower temperatures [16]. Consequently, concluded that the linear dextran is more viscous in nature rather than the branched dextran. Conversely, the dextransucrase of Weissella cibaria NITCSK4 favors the yield of low molecular weight dextran at a higher temperature $\left(35^{\circ} \mathrm{C}\right)$. The enzyme with low substrate concentration ( $3 \%$ sucrose) at $35^{\circ} \mathrm{C}$ produced higher amount of LMWD rather than HMWD, on the other hand the yield of total seems to be low. Moreover, the dextransucrase DSWc4 activity was virtuous in the temperature range $25-35^{\circ} \mathrm{C}$. However, at $45^{\circ} \mathrm{C}$, the DSWc4 produced a smaller amount dextran; this was due to the instability of the dextransucrase at a higher temperature

. Thus the dextransucrase of Weissella cibaria is stable up to $35^{\circ} \mathrm{C}$.

\section{F. Molecular Weight Distribution of LMWDexWe4}

The number average molecular weight of GPC fractions $(<100 \mathrm{kDa})$ obtained from the reaction mixture (validated condition) was determined. The LMW-DexWc4 obtained from the enzymatic conversion process was fractionated based on their size using gel permeation chromatography. The elution pattern of the standard dextran and LMW-DexWc4 is presented in Fig. 6.

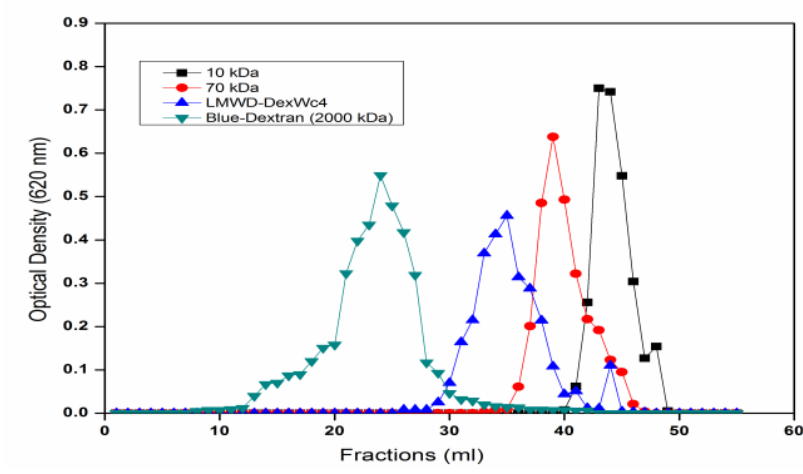

Fig. 6 GPC analysis of standard dextran (10 and 70 kDa), LMWD obtained from the dextransucrase of Weissella cibaria NITCSK4 (DexWc4), and blue dextran $(2000 \mathrm{kDa})$

The LMW-DexWc4 was precipitated with ice-cold ethanol and lyophilized. The number average molecular weight (MWn) of LMW-DexWc4 was determined and it was found to be $90 \mathrm{kDa}$. The water-soluble low molecular weight dextran solutions reported to behave as Newtonian fluids since the viscosity of dextran solution is influenced by the concentration, temperature and molecular weight [4].The intrinsic viscosity of LMW-DexWc4 was found to be $19.928\left(\mathrm{~cm}^{3} / \mathrm{g}\right)$. In 2013, Masuelli extensively and experimentally studied and reviewed the intrinsic viscosity measurements and temperature effect of dextran in aqueous solutions [14]. The SEM analysis of LMW-DexWc4 revealed solid rigid sphere morphology as shown in Fig. 7

\section{Table-IV: The Number Average and Weight Average Molecular Weight of Dextran}

\begin{tabular}{|l|l|l|l|}
\hline \multirow{3}{*}{ Dextran } & MWw (Da) & MWn (Da) & $\begin{array}{l}\text { \% Relative } \\
\text { Error }\end{array}$ \\
\hline \multirow{3}{*}{ Standard } & $10000^{*}$ & 10238 & 2.38 \\
\cline { 2 - 4 } & $70000^{*}$ & 71234 & 1.76 \\
\cline { 2 - 4 } & $5000000-40000000^{*}$ & 25344268 & - \\
\hline LMW-DexWc4 & 85775 & 89569 & 4.42 \\
\hline & \multicolumn{3}{|c|}{$\begin{array}{c}\text { *provided by Manufacturer, \# calculated } \\
\text { MWw-Weight average Molecular Weight } \\
\text { MWn-Number Average Molecular Weight }\end{array}$}
\end{tabular}
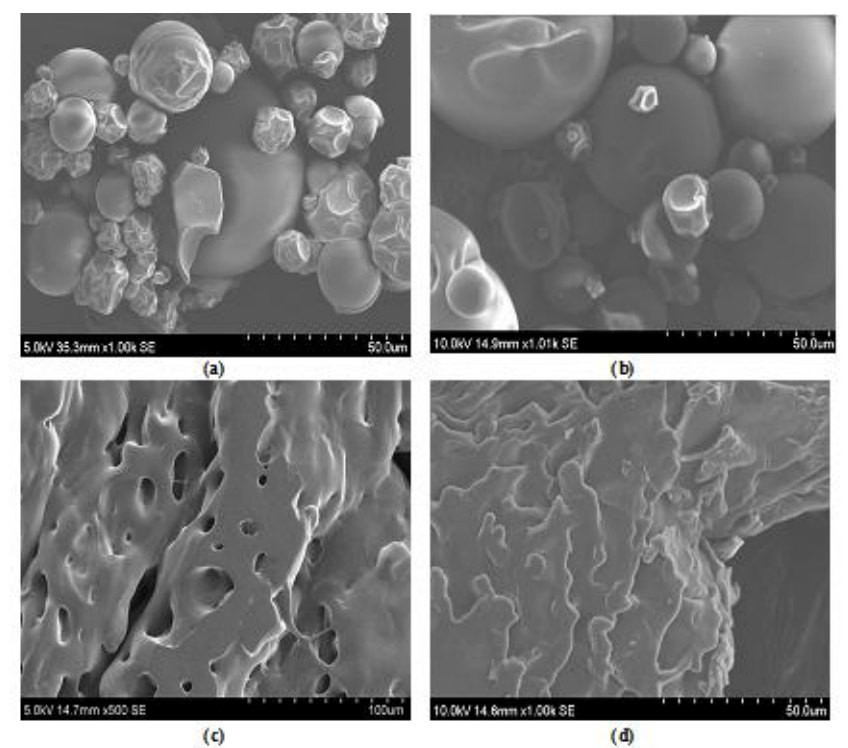

Fig.7 SEM images: (a). Standard dextran (70 kDa), (b). LMW-DexWc4 obtained from GPC fractions $(<100 \mathrm{kDa})$, (c). Standard HMW dextran (5000-40,000 kDa), (d).HMW-DexWc4.

The three-dimensional configuration of solid sphere reported bearing the Mark-Houwink exponent value of 0-0.5. Similarly, Mark Houwink parameter of dextran in water at $30^{\circ} \mathrm{C}$ for varied MW $(10-2000 \mathrm{kDa})$ was reported to be 0.4244 and $0.1606 \mathrm{~cm}^{3} / \mathrm{g}$ [14]. The mentioned values were assumed to calculate the weight average molecular weight of LMW-DexWc4 using Mark-Houwink expression (Eq. 6).The weight average molecular weight of LMW-DexWc4 was found to be $85775(\approx 90 \mathrm{kDa})$. 
The relative percentage error between the number and weight average molecular weight was found to be less $(4.42 \%)$ as shown in Table 4 . The polydispersity index (PDI) is the ratio of weight average molecular weight and number average molecular weight of polymer solution. The PDI of the LMW-DexWc4 was found to be 0.9576 and the value is close to 1 , the molecular weight distribution is narrow dispersed [14], [16].

\section{CONCLUSION}

The low molecular weight dextran of Weissella cibaria NITCSK4 (LMWD) could be prepared by controlling reaction conditions. In spite of the yield of low molecular weight dextran (LMWD), the overall dextran yield was found to be less by the dextransucrase of Weissella cibaria NITCSK4 (DSWc4) compared to microbial fermentative production of dextran. Owing to the consideration of downstream processing of preparation of LMWD from the hydrolysis of native dextran, the enzymatic conversion process could be adapted for the preparation of LMWD.

\section{REFERENCES}

1. M. Naessens, A. Cerdobbel, W. Soetaert, and E. J. Vandamme, "Leuconostocdextransucrase and dextran: production, properties and applications," J. Chem. Technol. Biotechnol., vol. 80, no. 8, pp. 845-860, Aug. 2005

2. R. Baruah, N. H. Maina, K. Katina, R. Juvonen, and A. Goyal, "Functional food applications of dextran from Weissella cibaria RBA12 from pummelo (Citrus maxima)," Int. J. Food Microbiol., vol. 242, pp. 124-131, 2016.

3. H. Leemhuis et al., "Glucansucrases: Three-dimensional structures, reactions, mechanism, $\alpha$-glucan analysis and their implications in biotechnology and food applications," J. Biotechnol., vol. 163, no. 2, pp. 250-272, 2013.

4. B. H. A. Rehm, "Bacterial polymers: Biosynthesis, modifications and applications," Nat. Rev. Microbiol., vol. 8, no. 8, pp. 578-592, 2010.

5. J. F. Robyt, S. H. Yoon, and R. Mukerjea, "Dextransucrase and the mechanism for dextran biosynthesis," Carbohydr. Res., vol. 343, no. 18, pp. 3039-3048, 2008.

6. K. C. Mountzouris, S. G. Gilmour, and R. A. Rastall, "Continuous production of oligodextrans via controlled hydrolysis of dextran in an enzyme membrane reactor," J. Food Sci., vol. 67, no. 5, pp. 1767-1771, 2002.

7. D. Kim, J. F. Robyt, S. Lee, J. Lee, and Y. Kim, "Dextran molecular size and degree of branching as a function of sucrose concentration, $\mathrm{pH}$, and temperature of reaction of Leuconostoc mesenteroides B-512FMCM dextransucrase,"Carbohydr.. Researc., vol. 338, pp. 1183-1189, 2003.

8. D. J. Falconer, R. Mukerjea, and J. F. Robyt, "Biosynthesis of dextrans with different molecular weights by selecting the concentration of Leuconostoc mesenteroides B-512FMC dextransucrase, the sucrose concentration, and the temperature," Carbohydr. Res., vol. 346, no. 2, pp. 280-284, 2011.

9. J. Kanimozhi, I. G. Moorthy, R. Sivashankar, and V. Sivasubramanian, "Optimization of dextran production by Weissella cibaria NITCSK4 using Response Surface Methodology-Genetic Algorithm based technology," Carbohydr. Polym., vol. 174, pp. 103-110, 2017.

10. S. Venkata Mohan and M. Venkateswar Reddy, "Optimization of critical factors to enhance polyhydroxyalkanoates synthesis by mixed culture using Taguchi design," Bioresour. Technol., vol. 128, pp. 409-416, 2013.

11. M. Parlak, D. Ustek, and A. Tanriseven, "A novel method for covalent immobilization of dextransucrase," J. Mol. Catal. B Enzym., vol. 89, pp. 52-60, 2013.

12. R. Pundir, G. H. V. C. Chary, and M. G. Dastidar, "Application of Taguchi method for optimizing the process parameters for the removal of copper and nickel by growing Aspergillus sp.," Water Resour. Ind., pp. 1-10, 2016.

13. M. Shanmugaprakash and V. Sivakumar, "Development of experimental design approach and ANN-based models for determination of $\mathrm{Cr}(\mathrm{VI})$ ions uptake rate from aqueous solution onto the solid biodiesel waste residue," Bioresour. Technol., vol. 148, pp. 550-559, 2013.

14. M. A. Masuelli, "Dextrans in Aqueous Solution: Experimental review on intrinsic viscosity measurements and temperature effect," J. Polym. Biopolym. Phys. Chem., vol. 1, no. 1, pp. 13-21, 2013.

15. M. Santos, J. Teixeira, and A. Rodrigues, "Production of dextransucrase, dextran and fructose from sucrose using Leuconostoc mesenteroides NRRL B512(f)," Biochem. Eng. J., vol. 4, no. 3, pp. 177-188, 2000.

16. M. Parlak, D. Ustek, and A. Tanriseven, "Designing of a novel dextransucrase efficient in acceptor reactions," Carbohydr. Res., vol. 386, no. 1, pp. 41-47, 2014. 